\title{
Classification of Suspected Liver Metastases Using fMRI Images: A Machine Learning Approach
}

\author{
M. Freiman ${ }^{1}$, Y. Edrei ${ }^{2,3}$, Y. Sela ${ }^{1}$, Y. Shmidmayer ${ }^{1}$, E. Gross $^{4}$, L. Joskowicz $^{1}$, \\ and R. Abramovitch ${ }^{2,3}$ \\ ${ }^{1}$ School of Eng. and Computer Science, The Hebrew Univ. of Jerusalem, Israel \\ ${ }^{2}$ The G. Savad Inst. for Gene Therapy, Hadassah Hebrew Univ. Medical Center, \\ Jerusalem, Israel \\ 3 MRI/MRS lab HBRC, Hadassah Hebrew Univ. Medical Center, Jerusalem, Israel \\ ${ }^{4}$ Pediatric Surgery, Hadassah Hebrew Univ. Medical Center, Jerusalem, Israel \\ freiman@cs.huji.ac.il
}

\begin{abstract}
This paper presents a machine-learning approach to the interactive classification of suspected liver metastases in fMRI images. The method uses fMRI-based statistical modeling to characterize colorectal hepatic metastases and follow their early hemodynamical changes. Changes in hepatic hemodynamics are evaluated from $T_{2}^{*}-\mathrm{W}$ fMRI images acquired during the breathing of air, air- $\mathrm{CO}_{2}$, and carbogen. A classification model is build to differentiate between tumors and healthy liver tissues. To validate our method, a model was built from 29 mice datasets, and used to classify suspicious regions in 16 new datasets of healthy subjects or subjects with metastases in earlier growth phases. Our experimental results on mice yielded an accuracy of $78 \%$ with high precision $(88 \%)$. This suggests that the method can provide a useful aid for early detection of liver metastases.
\end{abstract}

\section{Introduction}

The liver is the largest internal organ in the body, responsible for numerous metabolic, regulatory, transport, and immune functions. It is the second most commonly involved organ in metastatic disease, after the lymph nodes. The liver provides a fertile soil in which metastases can develop due to its rich, dual blood supply. In particular, hepatic metastases are a frequent complication in colorectal carcinoma patients. Since liver function tests in patients with liver metastases tend to be insensitive and non-specific, the disease is usually diagnosed at later stages of its development. Although numerous possible treatments are currently available, hepatic metastases are difficult to eradicate because they are discovered late. Early and accurate detection of these lesions has the potential of improving survival rates and reducing treatment morbidity.

A key physiological observation is that changes in liver blood supply can serve as an indicator for the presence of hepatic metastases [1. While the blood supply of healthy liver predominantly enters from the portal vein, in patients with overt 
colorectal liver metastases, a higher proportion of liver blood flow is derived from the hepatic artery. Thus, monitoring hemodynamical changes can serve as the basis for the early detection of hepatic metastases.

Medical imaging has the potential to play an important role in the monitoring of the liver hemodynamical changes. Barash et al 2 have recently demonstrated the utility of functional Magnetic Resonance Imaging (fMRI) with hypercapnia and hyperoxia for monitoring changes in liver perfusion and hemodynamics without contrast agent administration. Based on these findings, Edrei et al 3 showed how to characterize colorectal hepatic metastases and how to follow their early hemodynamical changes. Unlike methods that use contrast agents 415, their method can detect steady state levels without making a compromise between the spatial and temporal resolutions. This opens up the possibility to detect smaller lesions at an earlier development stage. Since hemodynamical changes are subtle, relative, and spatially distributed, direct observation of these changes is difficult, unreliable, and time-consuming.

Computer-aided detection of early hemodynamical changes from fMRI images is a challenging task. Existing methods for brain fMRI analysis, such as massmultivariate analysis 677 and region-based methods [89 cannot be applied to the liver. The analysis must cope with high inter-subject variability in the spatial locations of the metastases, their functional activation response, and the GRE (Gradient Recalled Echo) signal variations of the different tissues.

In previous work 10, we developed a method to classify manually selected and normalized rectangular regions on liver fMRI images as metastatic or healthy tissue. Our method uses a non-linear Support Vector Machine (SVM) [1] model constructed from manually selected samples of fMRI images taken at the advanced growth phase of the metastases. The drawbacks of this method are that it is operator-depended in region selection and normalization, and that it is biased to axis-aligned shapes, which are not conformal to metastases shapes.

This paper presents a machine-learning approach to the interactive classification of suspected liver metastases using fMRI images. The method uses fMRI-based statistical modeling to characterize colorectal hepatic metastases and follow their early hemodynamical changes. These changes are evaluated from $T_{2}^{*}-\mathrm{W}$ fMRI images acquired during the breathing of air, air- $\mathrm{CO}_{2}$, and carbogen. A classification model is build to differentiate between tumor and healthy liver tissues. The advantages of our classification model are that its construction does not require user interaction and and manual normalization. Thus, it is userindependent, and more accurate as it is not biased to axis-aligned shapes. The proposed model allows radiologists to interactively examine suspicious locations of early metastases presence in anatomical MRI images.

\section{Method}

Our method follows the supervised learning paradigm. First, it builds a classification model from a training set of image datasets of confirmed healthy subjects or subjects with confirmed metastases at the advanced growth phase. The model 
is then used to classify suspicious regions in new datasets of healthy subjects or subjects with metastases at earlier growth phases. Each dataset is processed with the same dataset analysis method.

The input dataset consists of an anatomical MRI image $\left(T_{2} \mathrm{~W}\right)$ and a set of fMRI-based maps representing hepatic hemodynamics. The fMRI images are acquired during the breathing of air, air-carbon dioxide $\left(5 \% \mathrm{CO}_{2}\right)$, and carbogen (95\% oxygen; $5 \% \mathrm{CO}_{2}$ ) according to the protocol in [2], and co-registered.

\subsection{Dataset Analysis}

Dataset analysis consists of four steps as follows.

\section{Hemodynamic activity maps computation}

Two hemodynamic activity maps are derived from the fMRI images. The maps describe the mean relative change of the fMRI signal intensity induced by hypercapnia $\left(\Delta S_{\mathrm{Co}_{2}}(\boldsymbol{x})\right)$ and hyperoxia $\left(\Delta S_{\mathrm{O}_{2}}(\boldsymbol{x})\right)$ :

$$
\Delta S_{\mathrm{CO}_{2}}(\boldsymbol{x})=\frac{\bar{S}_{\mathrm{co}_{2}}(\boldsymbol{x})-\bar{S}_{a i r}(\boldsymbol{x})}{\bar{S}_{\text {air }}(\boldsymbol{x})} \times 100 \quad \Delta S_{\mathrm{O}_{2}}(\boldsymbol{x})=\frac{\bar{S}_{\mathrm{O}_{2}}(\boldsymbol{x})-\bar{S}_{\mathrm{co}_{2}}(\boldsymbol{x})}{\bar{S}_{\mathrm{co}_{2}}(\boldsymbol{x})} \times 100
$$

where $\boldsymbol{x}$ is the pixel coordinates and $\bar{S}$ is the mean signal value over all acquisitions for each gas inhalation. The colorectal hepatic metastases are present in regions with reduced $\Delta S_{\mathrm{O}_{2}}$ positive values and reduced $\Delta S_{\mathrm{Co}_{2}}$ negative values.

\section{Maps normalization}

Normalization of the hemodynamic activity maps is necessary due to the high variability of the GRE signal from different subjects and in the same subject at different time points. First, the liver borders are determined from the anatomical MRI images and all the rest is eliminated from the maps. Next, each map is centered around a liver mean intensity of zero, with a standard deviation of 1 :

$$
\tilde{\Delta} S_{c o_{2}}(\boldsymbol{x})=\frac{\Delta S_{\mathrm{co}_{2}}(\boldsymbol{x})-\mu_{\Delta S_{\mathrm{co}_{2} h l}}}{\sigma_{\Delta S_{\mathrm{co}_{2} h l}}} \quad \tilde{\Delta} S_{O_{2}}(\boldsymbol{x})=\frac{\Delta S_{o_{2}}(\boldsymbol{x})-\mu_{\Delta S_{o_{2} h l}}}{\sigma_{\Delta S_{o_{2}} h}}
$$

where $\boldsymbol{x}$ is the pixel coordinates, $\mu_{\Delta S_{\mathrm{Co}_{2} h l}}$ and $\mu_{\Delta S_{o_{2} h l}}$ are the mean values of the healthy liver region, and $\sigma$ is their corresponding standard deviation. Finally, each dataset is filtered with bilateral smoothing [12] to reduce the amount of noise while keeping the discontinuities between healthy and metastasis regions.

\section{Maps adaptive partitioning}

The next step is the partition of the hemodynamic activity maps into regions based on spatial proximity and hemodynamic activity values. Adaptive automatic partition of the maps is necessary since the number, location, and shape of the metastases varies from subject to subject. This is in contrast to brain fMRI, where the regions of cognitive activity are similar and known in advance. We compute the spatial partition into regions of similar hemodynamic activity 


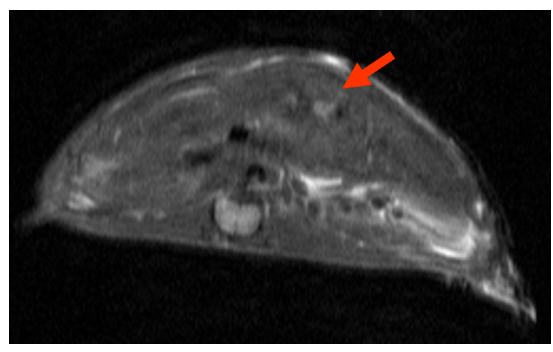

(a) Anatomical image

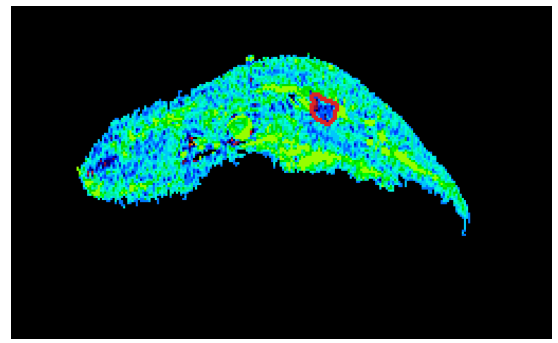

(c) $\Delta S_{\mathrm{Co}_{2}} \operatorname{map}$

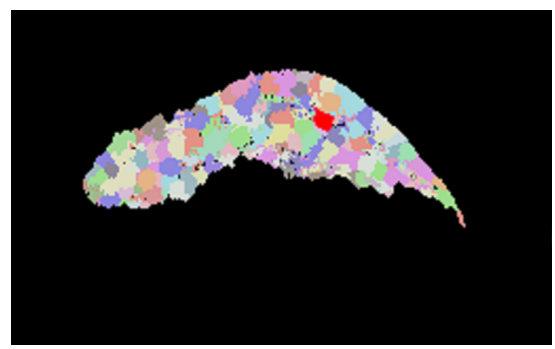

(b) Liver partitioned image

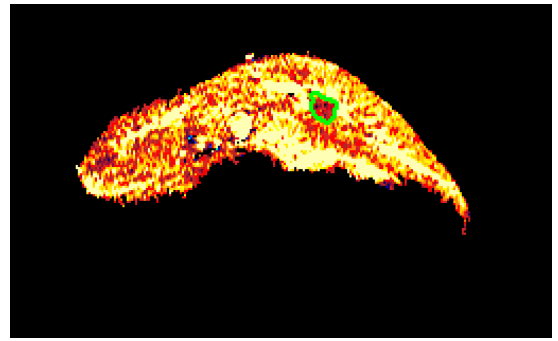

(d) $\Delta S_{o_{2}} \operatorname{map}$

Fig. 1. Illustration of early phase hemodynamic maps partitioning: (a) anatomical MRI image (the suspected metastatic region is not yet confirmed); (b) partition map in which each region has a different color; the suspicious region is colored by red; (c)-(d) hemodynamic activity maps showing the suspicious region. Note that the hemodynamic maps are presented after normalization

by mean-shift clustering [13] of both $\Delta S$ maps. Fig. 1] shows an example of the clustering results of an early phase dataset.

\section{Features extraction}

Next, we compute two characteristic feature vectors for each region $R_{k}$ in the partition. Since metastases have different locations, shapes, and sizes, the features vectors must be invariant to them. Since reactivity to hypercapnia and hyperoxia distinguishes between two different biological properties, we compute a separate feature vector for each map. We chose to compactly describe the regions distribution with a feature vector $\boldsymbol{f}_{k}$ consisting of five parameters: 1) mean; 2) standard deviation; 3) kurtosis; 4) skewness, and 5) interquartile range. This representation yields better results than using the entire histogram [10]. Fig. 2 shows two sample histograms of healthy and metastatic regions.

\subsection{Model Construction}

To generate the classification model of the metastases, we process the images in the training set as described above. The output is a set of regions and their feature vectors description. Each region is individually labeled by an expert radiologist as healthy/metastatic based on the anatomical MRI images. 


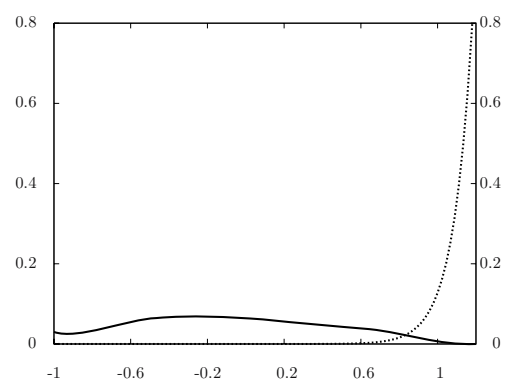

(a) Advanced $\tilde{\Delta} S_{\mathrm{Co}_{2}}$

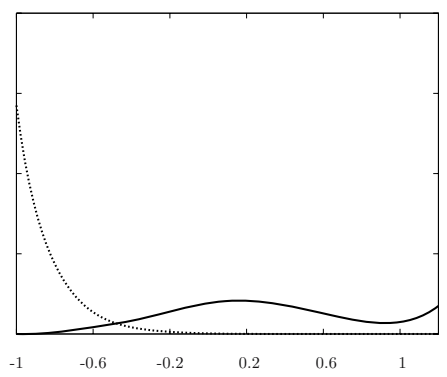

(b) Advanced $\tilde{\Delta} S_{o_{2}}$

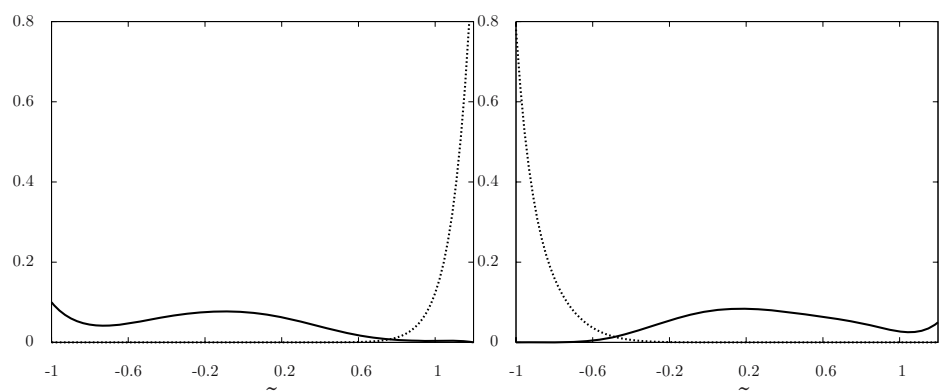

(c) Early $\tilde{\Delta} S_{\mathrm{Co}_{2}}$

(d) Early $\tilde{\Delta} S_{o_{2}}$

Fig. 2. Representative sample distributions of normalized hemodynamic activity maps of metastatic (dotted line) and healthy (solid line) regions: (a)-(b) advanced growth phase; (c)-(d) early growth phase

We train an SVM classification engine with the resulting set of tagged feature vector. We compute a classification model for each hemodynamic activity map separately. We use a generalized Radial Basis Function (RBF) kernel with the Earth Movers Distance (EMD) [14 as the affinity measure.

\subsection{Early Classification}

Once the classification model has been constructed, we use it to classify suspicious regions in new datasets of healthy subjects or subjects with metastases in earlier growth phases. The radiologist identifies a location of a suspected metastasis in the anatomical MRI. The corresponding region in the hemodynamic activity maps is then classified according to the classification model. This can be repeated for additional locations as required.

The dataset is processed following the dataset analysis method described above. The two hemodynamic activity maps are first computed and normalized. We use a truncated mean used instead of the standard mean in Eq. 2 to reduce the sensitivity to noise and to small metastases possibly present inside the liver. The maps adaptive partition is then performed on the resulting maps. The features extraction is then performed on the regions containing the locations selected by the radiologist. Since two different and independent biological 


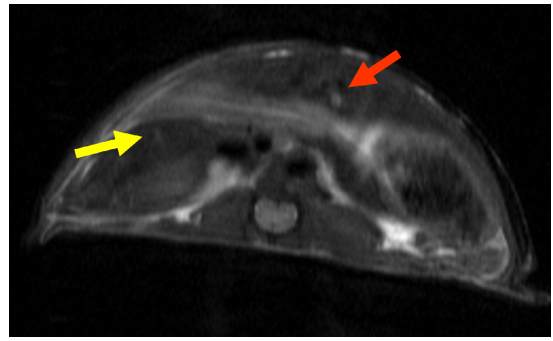

(a) Early phase

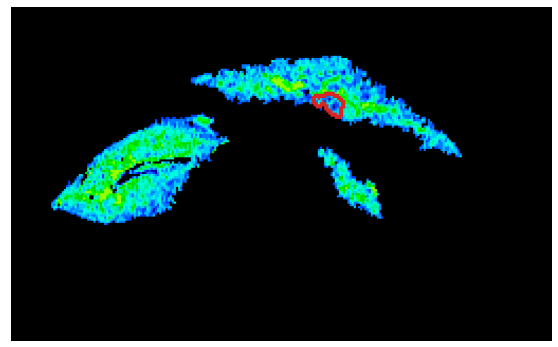

(c) $\Delta S_{\mathrm{Co}_{2}} \operatorname{map}$

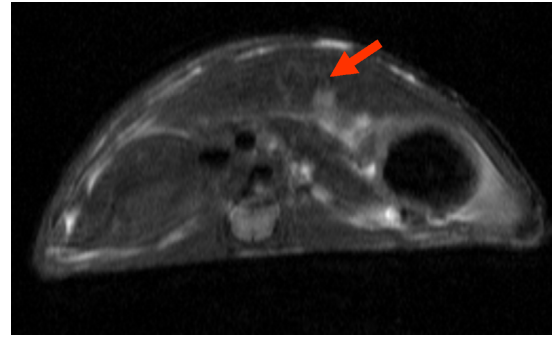

(b) Advanced phase

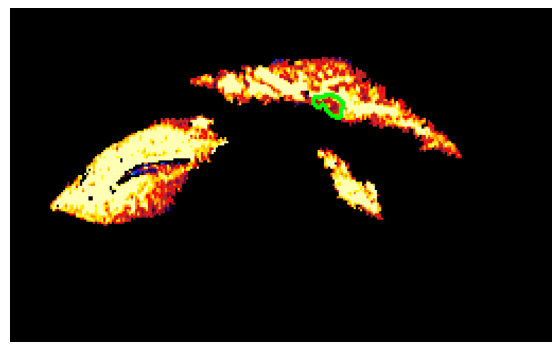

(d) $\Delta S_{o_{2}}$ map

Fig. 3. Early classification example: (a) anatomical MRI image with suspected regions pointed; (b) anatomical MRI image from advanced growth phase of the same subject. Only the region pointed by the red arrow was classified as metastasis by our method, as confirmed later; (c)-(d) early phase hemodynamic activity maps. The suspected region is marked. Although the anatomical images (a-b) are not co-registered since they are acquired at different times. The correspondence between the lesions in the images was determined by an expert using their position relative to the kidneys.

processes are modeled by the two maps, we classify the hemodynamic activity maps feature vectors separately and join the results. When both are classified as metastasis, the region is considered as metastatic. Coupling the classification yielded worse results than separate treatment. Fig. 3 shows an example of the early phase confirmation. The pointed areas are the suspected regions. Note that there are no confirmed metastases in the anatomical image (Fig. 3(a)), while the corresponding hemodynamical changes are easily detectable in the hemodynamic activity maps (Fig. $3(\mathrm{c})$ 3(d) . The region pointed by the red arrow was confirmed as containing a metastasis in the advanced growth phase anatomical image $(3(\mathrm{~b})$.

\section{Experimental Results}

We performed an animal study on CB6F1 mice that underwent splenic injection with CT-26 colon cancer cells $\left(10^{4}\right.$ cells $/$ mouse in $\left.0.3 \mathrm{ml}\right)$ to generate metastases. Metastases progression was monitored twice a week by MRI. MRI scans were performed on a $4.7 \mathrm{~T}$ Bruker Biospec spectrometer with a $3 \mathrm{~cm}$ bird cage 
Table 1. Summary of experimental results tabulated in a confusion matrix. The columns indicate the true class (Positive/Negative) and the rows indicate the hypothesized class (Yes/No).

\begin{tabular}{|c||c|c|}
\hline & Positive & Negative \\
\hline \hline Yes & 23 & 3 \\
\hline No & 7 & 11 \\
\hline \hline Total & 30 & 14 \\
\hline
\end{tabular}

coil. Anatomical MRI images were acquired using $T_{2}$-weighted fast SE images $(\mathrm{TR} / \mathrm{TE}=2000 / 40 \mathrm{~ms})$. Changes in hepatic hemodynamics were evaluated using $T_{2}^{*}$-weighted GRE (TR/TE $\left.=147 / 10 \mathrm{~ms}\right)$. The images were acquired following the protocol in [2] and co-registered since the mice are fixed and immobilized under anesthesia. When metastases became large (18 days after tumor cells injection, and above), the mice were sacrificed, and their livers were taken for histology.

A classification model was generated from 25 advanced growth phase (days 14-18) datasets and 4 of healthy subjects datasets. Each dataset consist of 4-5 slices with 1-5 metastases. Since only 4 slices are available for each mouse, real 3D analysis cannot be performed. Therefore each slice was analyzed separately. The model was generated with the SVM engine implementation from [15].

To test early classification, we obtained 16 early growth phase (days 10-14) datasets, and preprocessed them as described in Sec. 2.3. An expert radiologist selected a total of 44 suspected metastases locations on the anatomical images (the metastases were not confirmed in the anatomical MRI images). The corresponding regions in the hemodynamic activity maps were then classified and confirmed later by histology or by an additional advanced growth phase anatomical MRI. Table 1 summarizes the results. The classification performance of our method was $78 \%$ accuracy, $88 \%$ precision, and of $77 \%$ recall.

\section{Conclusions}

We have presented a new method for the interactive classification of suspected liver metastases using fMRI images. The method uses a machine-learning approach to construct an fMRI-based statistical model to characterize colorectal hepatic metastases from a training dataset. The classification model is then used to classify suspicious regions in new datasets of healthy subjects or subjects with metastases in earlier growth phases. Our experimental results on mice yielded an accuracy of $78 \%$ with high precision (88\%). This suggests that the method can provide a useful aid for early detection of metastases. We are conducting ongoing research to determine the applicability of our method to humans.

Financial support: This research was supported in part by a grant number 728/05 from the Israel Science Foundation (for RA), by the Horwitz Foundation through The Center for Complexity Science (for RA and YE). 


\section{References}

1. Leveson, S., Wiggins, P., Giles, G., Parkin, A., Robinson, P.: Deranged blood flow patterns in the detection of liver metastases. Bri. J. of Surgery 72, 128-130 (1985)

2. Barash, H., Gross, E., Matot, I., Edrei, Y., Tsarfaty, G., Spira, G., Vlodavsky, I., Galun, E., Abramovitch, R.: Functional-MRI during hypercapnia and hyperoxia: a non-invasive monitoring tool for changes in liver perfusion and hemodynamics in a rat model. Radiology (243), 727-735 (2007)

3. Edrei, Y., Gross, E., Pikarsky, E., Galun, E., Abramovitch, R.: Characterization and early detection of liver metastasis by fMRI. In: The 14th Int. Soc. of Magnetic Res. in Medicine (ISMRM) Scientific Meeting (2006) (Abstract no. 1752)

4. Cuenod, C., Leconte, I., Siauve, N., Resten, A., Dromain, C., Poulet, B., Frouin, F., Clement, O., Frija, G.: Early changes in liver perfusion caused by occult metastases in rats: Detection with quantitative CT. Radiology 218(2), 556-561 (2001)

5. Totman, J., O'Gorman, R., Kane, P., Karani, J.: Comparison of the hepatic perfusion index measured with gadolinium-enhanced volumetric MRI in controls and in patients with colorectal cancer. British J. of Radiology 78(926), 105-109 (2005)

6. Mitchell, T., Hutchinson, R., Niculescu, R., Pereira, F., Wang, X., Just, M., Newman, S.: Learning to decode cognitive states from brain images. Machine Learning 57(1-2), 145-175 (2004)

7. Wang, X., Hutchinson, R., Mitchell, T.: Training fMRI classifiers to discriminate cognitive states across multiple subjects. In: Advances in Neural Inf. Processing Systems, vol. 16. MIT Press, Cambridge (2004)

8. Thirion, B., Faugeras, O.: Feature characterization in fmri data: the information bottleneck approach. Med. Image Analysis 8(4), 403-419 (2004)

9. Fan, Y., Shen, D., Davatzikos, C.: Detecting cognitive states from fmri images by machine learning and multivariate classification. In: Proc. of the Conf. on Comp. Vision and Patt. Recognition Workshop (CVPRW 2006), p. 89 (2006)

10. Freiman, M., Edrei, Y., Gross, E., Joskowicz, L., Abramovitch, R.: Liver metastases early detection using fMRI based statistical model. In: Proc. of the 5th IEEE Int. Symp. on Biomedical Imaging: From Nano to Macro (ISBI 2008) (2008)

11. Vapnik, V.: The nature of statistical learning theory. Springer, Heidelberg (1995)

12. Tomasi, C., Manduchi, R.: Bilateral filtering for gray and color images. In: Proc. of the 6th Int. Conf. on Comp. Vision (ICCV 1998), pp. 839-846 (1998)

13. Comaniciu, D., Meer, P.: Mean shift: a robust approach toward feature space analysis. IEEE Trans. Patt. Anal. and Mach. Intell. 24(5), 603-619 (2002)

14. Rubner, Y., Tomasi, C., Guibas, L.: A metric for distributions with applications to image databases. In: 6th Int. Conf. on Comp. Vision, ICCV, pp. 59-66 (1998)

15. Thorsten, J.: Making large-scale support vector machine learning practical. In: Schölkopf, B., Burges, C., Smola, A. (eds.) Advances in kernel methods: support vector learning, pp. 169-184. MIT Press, Cambridge (1999) 